\title{
LA REACTION CORTICOSURRÉNALE LORS D'UNE TENTATIVE DE RÉINFESTATION DES RATS PAR STRONGYLOIDES RATTI
}

\author{
par J. BAILENGER, J. B. CHANRAUD et A. CABANNES*
}

\begin{abstract}
RÉSUMÉ. Les tentatives de réinfestation, effectuées après la guérison spontanée d'un parasitisme par Strongyloïdes ratti, ne réussissent pas à établir une nouvelle génération d'adultes lorsque la contamination primaire est réalisé par 5000 ou 10000 larves, à plusieurs reprises ou en une seule fois, et que la période de repos entre le déparasitage et la contamination secondaire est limitée à 3 semaines. La réinfestation commence à devenir possible après un repos de 51 jours suivant la guérison d'une infestation primaire par 15000 larves.

Mais, bien qu'elles n'atteignent pas l'état adulte, les larves infestantes commencent leur migration tissulaire et provoquent une hypersécrétion de gluco-cortico-stérö̈des.

Il existe donc une opposition entre la facilité avec laquelle l'organisme empêche, précocement et avec persistance, la transformation des larves en adultes et la difficulté qu'il éprouve à neutraliser les larves et à les empêcher de déclencher une agression.
\end{abstract}

\section{Cortico-adrenal reaction in rats to attempts of reinfestation by Strongyloides ratti.}

SUMMARY. After spontaneous recovery from infestation by Strongyloides ratti, reinfestation by a new generation of adult parasites is not possible if the primary infestation was by 5,000 or 10,000 larvae administered in one or more contacts and if the secondary infestation takes place 3 weeks after. Reinfestation becomes possible again 51 days after recovery from a primary infestation by 15,000 larvae.

However although the larvae do not reach the adult stage, they do begin their migration and cause hypersecretion of gluco-cortico-steroids.

There is thus a contrast between the ease with which the organism rapidly and lastingly prevents the transformation of larvae into adults and the difficulty in neutralising the larvae and preventing them from triggering a stress.

Dans une étude sur les variations de l'hypercorticostéronémie réactionnelle lors d'infestations répétées du Rat, par Strongyloides ratti (1980) nous avons montré que l'organisme, au sein duquel l'évolution du parasite est en cours, réagit à l'agression de nouvelles larves par une hypercorticostéronémie, malgré qu'il ne leur permette

- Laboratoire de Zoologie et Biologie animale, U.E.R. Pharmacie, Université Bordeaux II, Place de la Victoire, F 33076 Bordeaux 
pas d'atteindre l'état adulte. Cette réaction s'atténue très lentement avec la répétition des infestations et finit par ne phus se produire.

Après la cessation des infestations et la guérison spontanée, on peut se demander quel peut être le comportement de l'organisme mis au contact, une nouvelle fois, avec des larves infestantes.

Répondre à cette question est le but de ce travail. Cette réponse doit tenir compte des modalités de la primo-infestation : intensité ; infestation unique ou répétée. Elle doit également considérer le laps de temps écoulé entre le déparasitage et l'infestation secondaire.

Nous avons retenu des infestations primaires par 5000 et 10000 larves comportant une contamination unique ou multiple. La période qui s'écoule entre le déparasitage et la tentative de réinfestation varie de 3 jours à 25 jours.

\section{Matériel et méthodes}

Rats : mâles ; adultes ; 180 à $200 \mathrm{~g}$ au début de l'expérience, souche wistar : pathogenfree.

Infestations : balnéations individuelles de 50 minutes dans $90 \mathrm{ml}$ d'eau contenant un nombre déterminé de larves.

Parasitisme: Lors de l'infestation primaire, il est suivi par la numération des œufs et larves de St. ratti. Pour l'infestation secondaire, on pratique le coprologie jusqu'au huitième jour, puis on sacrifie les animaux et on examine la muqueuse de l'intestin grêle.

Corticostéronémie : Prélèvements sanguins à 9 heures; dosages par fluorescimétrie selon la méthode de De Moor avec la rectification de Moncloa telle que nous l'avons précédemment décrite (1974).

Pour chaque expérience, les rats sont groupés par six, en un nombre de lots défini par les conditions expérimentales. Tous les animaux sont soumis, selon des modalités rigoureusement identiques, à la primo-infestation puis à la tentative de ré-infestation. Les lots destinés au dosage de la corticostéronémie sont exclus de l'expérience après avoir subi le prélèvement sanguin, pour éviter les répercussions éventuelles de l'agression provoquée par cette intervention.

Des animaux non infestés la première fois, mais élevés dans les mêmes conditions que les autres, sont également soumis à l'infestation secondaire afin d'évaluer le pouvoir infestant des larves et l'agression qu'elles constituent pour un organisme neuf.

La réaction du cortex surrénal est établie par comparaison avec des animaux de même âge, élevés et manipulés rigoureusement dans les mêmes conditions, mais non infestés.

Chaque dosage de la corticostéronémie est effectué sur un échantillon de plasma 
correspondant au mélange de volumes égaux du plasma de tous les animaux d'un même lot. La valeur ainsi obtenue représente une moyenne pour tous les animaux de ce lot.

\section{Résultats}

1) Infestation primaire unique par 5 ooo larves; infestation secondaire par 6 ooo larves, 3 jours après le déparasitage (tableau I).

L'infestation secondaire, qui se place juste après le déparasitage, se situe 28 jours après la première contamination. Elle déclenche une hypercorticostéronémie $(59,3 \mu \mathrm{g})$ dont l'intensité est égale à celle que l'on note $(62,5 \mu \mathrm{g})$ chez les rats infestés pour la première fois. Par contre, tandis que ceux-ci sont normalement parasités, les rats précédemment infestés ne permettent pas une nouvelle installation des vers.

TABLEAU I. - Infestation primaire unique par 5000 larves Infestation secondaire par 6000 larves, 3 jours après le déparasitage

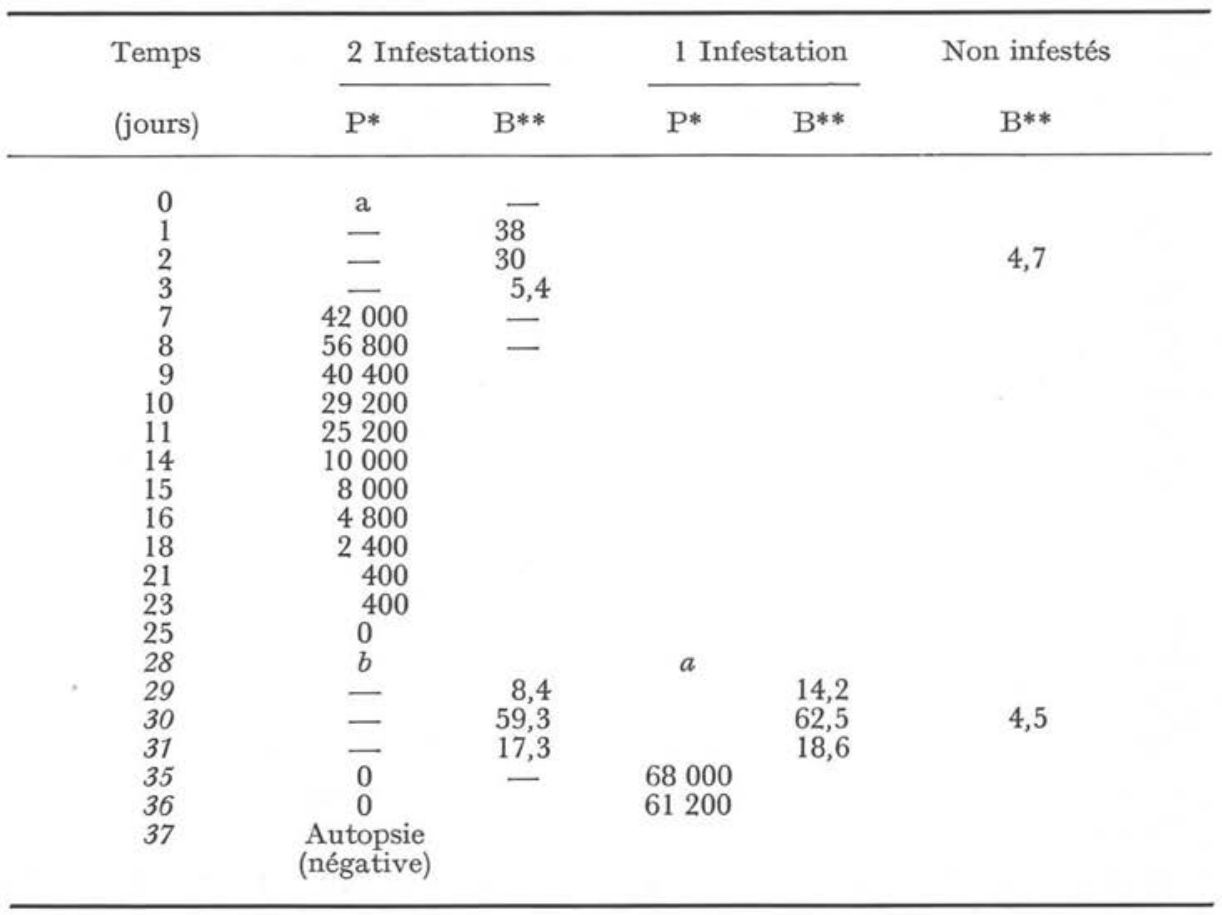

$\mathrm{P}^{*}$ : Parasitisme (œufs et larves par $\mathrm{g}$ de selles) ; B** : Corticostéronémie $(\mu \mathrm{g} / 100 \mathrm{ml})$.

$a$ : Infestation primaire ; $b$ : réinfestation. 
2) Infestation primaire multiple par 5000 larves; infestation secondaire par I2 000 larves, Io jours après le déparasitage (tableau II).

L'infestation primaire compte 6 infestations espacées de 2 à 3 jours, la dernière ayant lieu le $11^{\mathrm{e}}$ jour. La réinfestation se situe 10 jours après le déparasitage total, soit 26 jours après la dernière infestation primaire. Elle provoque une hypercorticostéronémie à $25,7 \mu \mathrm{g}$ comparable à celle des rats pour qui il s'agit d'une primoinfection $(35,7 \mu \mathrm{g})$. Le taux plasmatique de la corticostérone des témoins non parasités est de $4,5 \mu \mathrm{g}$.

Cette nouvelle réinfestation n'entraîne pas l'installation d'une nouvelle génération parasitaire.

TABLEAU II. - Infestation primaire multiple par 5000 lavves

Infestation secondaire par 12000 larves, 10 jours après le déparasitage

\begin{tabular}{|c|c|c|c|c|c|c|}
\hline \multirow[b]{2}{*}{$\begin{array}{l}\text { Temps } \\
\text { (jours) }\end{array}$} & \multicolumn{3}{|c|}{ Infestations primaire et secondaire } & \multicolumn{2}{|c|}{1 Infestation } & \multirow{2}{*}{$\begin{array}{l}\text { Non infestés } \\
\text { B** }\end{array}$} \\
\hline & $\begin{array}{l}\text { Infestations } \\
\text { Nbre }\end{array}$ & $\mathrm{P}^{*}$ & $\mathrm{~B} * *$ & $\mathrm{P}^{*}$ & $\mathrm{~B} * *$ & \\
\hline $\begin{array}{l}0 \\
1\end{array}$ & 1 & & & & & \\
\hline 2 & 2 & & 19,9 & & & \\
\hline $\begin{array}{l}3 \\
4\end{array}$ & 3 & & 11,6 & & & 4,7 \\
\hline 7 & 4 & 52400 & & & & \\
\hline 8 & & 83600 & & & & \\
\hline 9 & 5 & 77200 & 14,5 & & & \\
\hline $\begin{array}{l}10 \\
11\end{array}$ & 6 & $\begin{array}{ll}82 & 000 \\
74 & 000\end{array}$ & 16,6 & & & 5,7 \\
\hline 14 & & 21200 & 16,6 & & & \\
\hline $\begin{array}{l}15 \\
16\end{array}$ & & $\begin{array}{r}14000 \\
7600\end{array}$ & & & & \\
\hline 18 & & $\begin{array}{l}7600 \\
4400\end{array}$ & & & & \\
\hline 21 & & 1200 & & & & \\
\hline 23 & & 800 & & & & \\
\hline 25 & & 400 & & & & \\
\hline 37 & $b$ & & & $a$ & & \\
\hline 39 & & & 25,7 & & 35,7 & 4,5 \\
\hline 42 & & 0 & & 10400 & & \\
\hline 43 & & 0 & & 42000 & & \\
\hline 44 & & 0 & & 84800 & & \\
\hline 45 & & $\begin{array}{l}\text { Autopsie } \\
\text { (négative) }\end{array}$ & & 65200 & & \\
\hline
\end{tabular}

$a:$ Infestation primaire ; $b$ : réinfestation

* : Parasitisme (nombre d'œufs et de larves par g de selles) ; B** : Corticostéronémie $(\mu \mathrm{g} / 100 \mathrm{ml})$.

3) Infestation primaire multiple par Io ooo larves; infestation secondaire par I2 OOO larves, 25 jours après le déparasitage. 
L'infestation primaire est réalisée selon deux modalités; soit en deux fois, à quarante-huit heures d'intervalle (tableau III), soit en huit fois étalées sur 16 jours (tableau IV).

Dans les deux cas, la tentative de réinfestation a lieu vingt-cinq jours après le déparasitage. Elle ne conduit pas à un nouvel état parasitaire, mais elle déclenche toujours une hypercorticostéronémie qui est cependant plus atténuée chez les rats soumis à huit infestations primaires : chez les rats qui n'ont jamais encore été infestés la corticostéronémie monte à $40 \mu \mathrm{g}$, contre $2,9 \mu \mathrm{g}$ pour les témoins non infestés ; comparativement le taux de cette hormone est de $28,1 \mu \mathrm{g}$ pour les animaux ayant subi deux infestations primaires, tandis qu'il est de $14,1 \mu \mathrm{g}$ lorsque les contaminations primaires sont multiples.

TABLEAU III. - 2 Infestations primaires par 10000 larves Infestation secondaire par 12000 larves, 25 jours après le déparasitage

\begin{tabular}{|c|c|c|c|c|c|}
\hline \multirow{2}{*}{$\begin{array}{l}\text { Temps } \\
\text { (jours) }\end{array}$} & \multicolumn{2}{|c|}{ Infestations primaire et secondaire } & \multicolumn{2}{|c|}{1 Infestation } & \multirow{2}{*}{$\begin{array}{c}\text { Pas d'infestation } \\
\text { B** }\end{array}$} \\
\hline & $\mathrm{P}^{*}$ & $\mathrm{~B} * *$ & $\mathrm{P}^{*}$ & B** & \\
\hline $\begin{array}{r}0 \\
2 \\
7 \\
8 \\
9 \\
10 \\
11 \\
14 \\
15 \\
16 \\
17 \\
18 \\
21 \\
23 \\
48 \\
50 \\
55 \\
56 \\
57\end{array}$ & $\begin{array}{c}\mathrm{a} \\
\mathrm{a} \\
124800 \\
130400 \\
124000 \\
86400 \\
68400 \\
22000 \\
16000 \\
8400 \\
5600 \\
3200 \\
800 \\
0 \\
b \\
0 \\
0 \\
\text { Autopsie } \\
\text { (négative) }\end{array}$ & 28,1 & $\begin{array}{c}a \\
108000 \\
120000 \\
80800\end{array}$ & 40 & $\begin{array}{l}3,7 \\
2,7\end{array}$ \\
\hline
\end{tabular}

a : Infestation primaire; $\mathrm{b}$ : réinfestation.

$\mathrm{P}^{*}$ : Parasitisme (nombre d'œufs et de larves par g de selles) ; B** Corticostéronémie $(\mu \mathrm{g} / 100 \mathrm{ml})$.

4) Infestation primaire unique par I5 000 larves; infestation secondaire par I5 000 larves, 5 I jours après le déparasitage.

L'objectif de cette expérience complémentaire est limitée à l'exploration des possibilités de réinfestation. 
TABLEAU IV. - Infestation primaire multiple par 10000 larves

Infestation secondaive par 12000 larves, 23 jours après le déparasitage

\begin{tabular}{|c|c|c|c|c|c|c|}
\hline \multirow{2}{*}{$\begin{array}{l}\text { Temps } \\
\text { (jours) }\end{array}$} & \multicolumn{3}{|c|}{ Infestations primaire et secondaire } & \multicolumn{2}{|c|}{1 Infestation } & \multirow{2}{*}{$\begin{array}{c}\text { Pas d'infestation } \\
\qquad \mathrm{B}^{* *}\end{array}$} \\
\hline & Infestations & $\mathrm{P}^{*}$ & $\mathrm{~B}^{* *}$ & $\mathrm{P}^{*}$ & $\mathrm{~B}^{* *}$ & \\
\hline 0 & 1 & & - & & & \\
\hline 2 & 2 & & 22,2 & & & 2,9 \\
\hline 4 & 3 & & 12,5 & & & \\
\hline 7 & 4 & 146000 & & & & \\
\hline 8 & 二 & 144000 & & & & \\
\hline 9 & 5 & 136000 & & & & \\
\hline 10 & - & 82000 & & & & \\
\hline 11 & 6 & 78000 & & & & \\
\hline 14 & 7 & 51600 & & & & \\
\hline 15 & - & 24400 & & & & \\
\hline 16 & 8 & 14000 & 2,1 & & & 2,7 \\
\hline 17 & & 12000 & & & & \\
\hline 18 & & 4600 & & & & \\
\hline 21 & & 1200 & & & & \\
\hline 23 & & 800 & & & & \\
\hline 25 & & 0 & & & & \\
\hline 48 & b & & & a & & \\
\hline 50 & & & 14,1 & & 40 & 2,9 \\
\hline 55 & & 0 & & 108000 & & \\
\hline 56 & & 0 & & 120800 & & \\
\hline 57 & & $\begin{array}{c}\text { Autopsie } \\
\text { (négative) }\end{array}$ & & 80800 & & \\
\hline
\end{tabular}

a : Infestation primaire; $\mathrm{b}$ : réinfestation.

P* : Parasitisme (nombre d'œufs et de larves par g de selles) ;

$\mathrm{B}^{* *}$ : Corticostéronémie $(\mu \mathrm{g} / 100 \mathrm{ml})$.

En allongeant à 51 jours le laps de temps entre le déparasitage et l'infestation secondaire, l'examen coprologique révèle l'apparition d'un nombre très faible d'œufs et larves, tandis que les témoins correspondant à une primo-infestation, destinés à évaluer le pouvoir infestant des larves, éliminent au 7e jour 195000 éléments par g de selles. L'observation des rares éléments parasitaires dans les selles des rats réinfestés est fugace, puisqu'elle n'est positive qu'au septième jour et qu'elle se négative dès le lendemain. Néanmoins, cela suffit à affirmer qu'une réinfestation, bien que de densité et de durée réduites, devient possible après une infestation unique et forte, en allongeant à 51 jours le repos après déparasitage.

5) Infestation primaire unique par I5 ooo larves; infestation secondaire 7o jours après le déparasitage (tableau $V$ ).

Cette expérience confirme la possibilité, pour un petit nombre de vers, d'atteindre leur maturité sexuelle après un repos de 70 jours comptés à partir du déparasitage de la primo-infestation. 
Par comparaison avec l'expérience précédente pour laquelle le repos n'était que de 51 jours, le parasitisme s'intensifie et les vers adultes se maintiennent plus longtemps.

TABLEAU V. - Infestation primaire unique par 15000 larves

Réinfestation 70 jours après le déparasitage

\begin{tabular}{|c|c|c|}
\hline \multirow{2}{*}{$\begin{array}{l}\text { Temps } \\
\text { (jours) }\end{array}$} & \multicolumn{2}{|c|}{ Parasitisme (œufs et larves / $\mathrm{g}$ de selles) } \\
\hline & Infestation primaire & Témoins \\
\hline 5 & 152400 & - \\
\hline 7 & 289200 & - \\
\hline 9 & 231000 & - \\
\hline 11 & 126800 & - \\
\hline 14 & 24400 & - \\
\hline 16 & 3900 & - \\
\hline 18 & 600 & - \\
\hline 21 & 300 & - \\
\hline 23 & & - \\
\hline & Repos de 70 jours & \\
\hline 5 & $\begin{array}{c}\text { Réinfestations } \\
0\end{array}$ & 11200 \\
\hline 6 & 2700 & 11200 \\
\hline 7 & 1200 & 195000 \\
\hline 8 & 300 & - \\
\hline 9 & 0 & 93000 \\
\hline
\end{tabular}

\section{Discussion}

Nous avons retenu trois modalités expérimentales en faisant varier l'intensité des infestations (5000 et 10000 larves), leur répétition (contamination unique ou multiple) et la période de repos entre le déparasitage et l'infestation secondaire (3, 10 et 25 jours).

La réinfestation est impossible lorsque la contamination primaire est réalisée avec 5000 ou 10000 larves, à plusieurs reprises ou en une seule fois, et lorsque la période de repos entre le déparasitage et la contamination secondaire est limitée à 10 jours ou à 25 jours pour des infections primaires correspondant respectivement à 5000 et 10000 larves.

L'immunité qui s'est développée lors de l'infection primaire est donc suffisante pour s'opposer à l'implantation d'une nouvelle génération de Strongles adultes. Ce résultat correspond à ceux obtenus avec Strongyloides ratti (Sheldon 1937) ou Nippostrongylus brasiliensis (Jarrett et Urquhart 1968). Ces auteurs ont examiné les intestins des rats réinfestés dans des conditions voisines de celles que nous avons réalisées; ils notent que les vers qui ont réussi à s'installer dans l'intestin sont peu nombreux, qu'ils sont expulsés précocement et rapidement sans parvenir à leur maturité sexuelle. 
Cette immunité n'est cependant pas définitive. Elle disparaît progressivement puisqu'une réinfestation commence à devenir possible après un repos de 51 jours suivant la guérison d'une infestation primaire unique par 15000 larves et s'intensifie lorsque le laps de temps qui s'écoule entre le déparasitage et la réinfestation est porté à 70 jours.

Toutefois, pendant la période où l'organisme dispose d'une défense suffisamment efficace pour s'opposer à l'implantation des vers adultes, il ne réussit pas à empêcher les larves de déclencher une agression à laquelle il répond par une hypersécrétion de gluco-corticostéroïdes.

Nous avons précédemment mis en évidence (1980) que l'organisme acquiert très difficilement, à l'issue d'infestations répétées, cette possibilité de neutraliser les larves dès leur pénétration. En mesurant l'hypersécrétion cortico-surrénale qui évalue l'agression subie par l'organisme, nous montrons, dans ce travail, qu'il ne conserve cette faculté de neutraliser précocement les larves que pendant très peu de temps (fig. I). En effet, après guérison depuis 3 jours d'une infection primaire réalisée par un seul contact avec 5000 larves ou bien depuis 10 jours d'une infestation effectuée par 6 bains contaminants de 5000 larves, la réaction cortico-surrénale à une tentative de réinfestation est identique à celle d'animaux neufs. Lorsque la contamination secondaire se place 25 jours après le déparasitage d'une infestation primaire par 10000 larves, l'hypercorticostéronémie des rats infestés primitivement à deux reprises en deux jours est légèrement atténuée : $(28,1 \mu \mathrm{g}$ contre $40 \mu \mathrm{g}$ pour les animaux neufs). Si l'infestation primaire comporte 8 contaminations en 16 jours, la réaction corticosurrénale est nettement amoindrie $(14,7 \mu \mathrm{g}$ contre $40 \mu \mathrm{g})$ tout en existant, puisque la corticostéronémie d'animaux témoins non infestés est de $2,9 \mu \mathrm{g}$. Dans cette dernière expérience, la neutralisation des larves, telle qu'elles ne soient plus à l'origine d'une agression, n'a été acquise qu'après sept contaminations étalées sur 14 jours, c'est-àdire 32 jours seulement avant la réinfestation.

Ainsi, bien qu'elles ne donnent pas lieu à un état parasitaire dans un organisme guéri d'une infection passée, les formes infestantes d'un ver peuvent néanmoins déterminer une agression et ceci très rapidement après la guérison.

Cette agression traduit la persistance d'une migration larvaire soulignée par Jarrett et Urquhart (1968), Moqbel et Denham (1977) et particulièrement étudiée par Taliaferro et Sarles (1939) avec N. muris. Parmi les résultats publiés par ces derniers auteurs, nous retenons ceux correspondant à une infestation et à une réinfestation selon des modalités qui se rapprochent le plus des conditions expérimentales dans lesquelles nous nous sommes placés : réinfestation par 5000 larves, 6 à 8 semaines après 3 infestations à 3 jours d'intervalle par 5000 larves. Lors de la réinfestation on note une progression des larves de la peau vers les poumons, accompagnée d'une réaction inflammatoire plus précoce, plus intense et plus prolongée que chez les animaux neufs. Celle-ci peut aboutir à la formation de modules autour des larves les empêchant ainsi d'atteindre l'intestin. Seules quelques-unes y parviennent mais elles sont incapables de se développer normalement. 


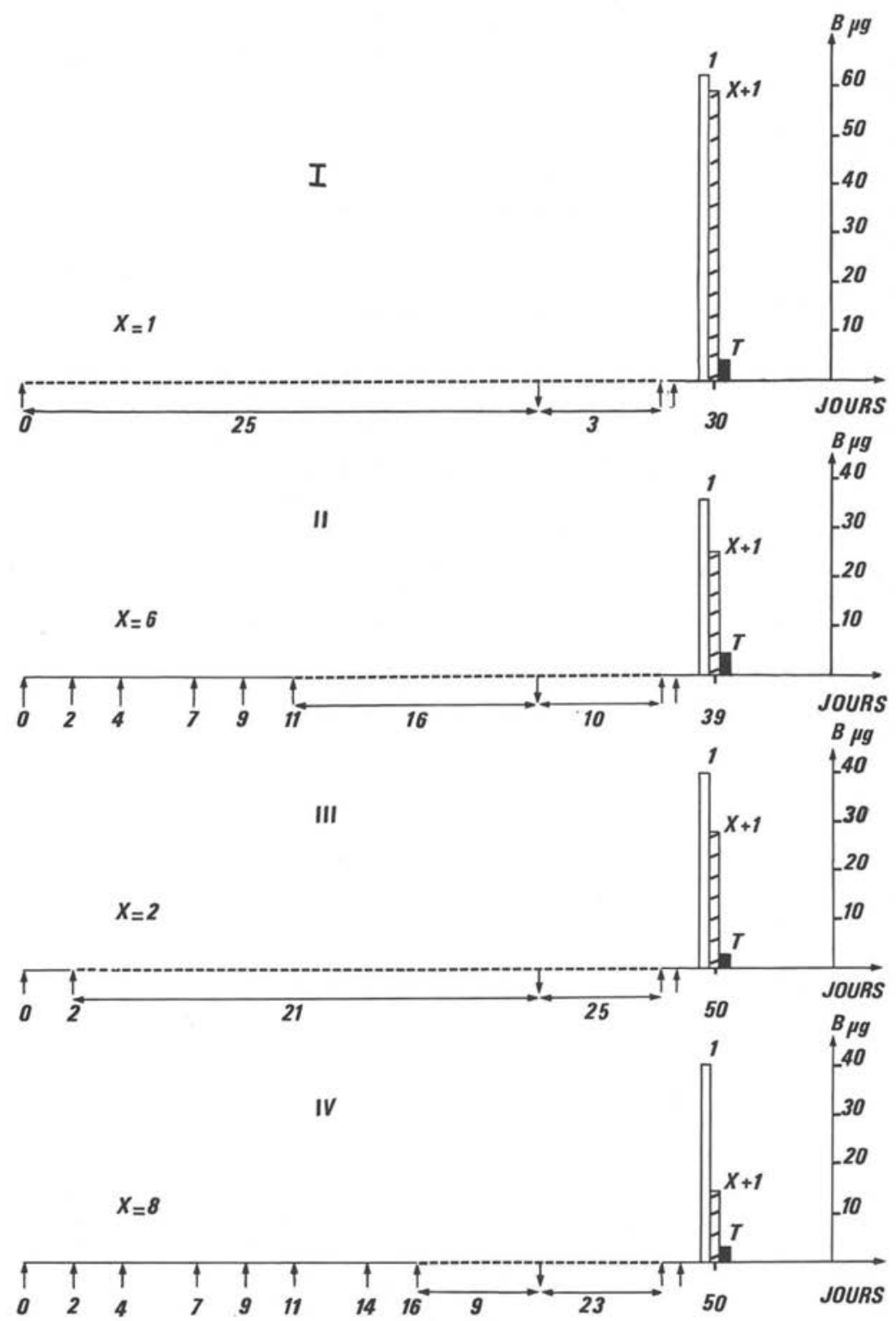

FIG. I. - Corticostéronémie $4^{8}$ heures après une tentative de réinfestation : variations en fonction des conditions de l'infestation primaire et de la durée du déparasitage.

B : Corticostéronémie ( $\mu \mathrm{g} / \mathrm{Ioo} \mathrm{ml})$.

$\uparrow:$ Infestations primaires ; $\downarrow$ déparasitage ; $\uparrow \uparrow$ réinfestation.

$\mathrm{T}$ : rats non infestés ; $\mathrm{I}$ : rats soumis à une seule infestation ; $\mathrm{X}$ : nombre d'infestations primaires.

Nombre de larves des infestations primaires : I : $5, \mathrm{IO}^{3} ; \mathrm{II}: 5,10^{3}$; III : $10, \mathrm{IO}^{3}$; IV : $10, \mathrm{IO}^{3}$. 
La réaction corticosurrénale que nous mettons en évidence objective la persistance de la progression tissulaire des larves lors d'une tentative de réinfestation d'un organisme en état d'immunité.

Remerciements. La souche de Strongyloides ratti est entretenue au Laboratoire d'Immunologie et Biologie parasitaire (Université Bordeaux II).

Nous remercions MM. les Professeurs Pautrizel et Tribouley d'avoir bien voulu la mettre à notre disposition.

\section{BIBLIOGRAPHIE}

Bailenger J., Chanraud J. B., Marcel P., Cabannes A. : Évolution du parasitisme et de la corticostéronémie lors d'infestations répétées des rats par Strongyloides ratti. Ann. Parasitol. Hum. Comp., 1981. s6, 317-327.

JARRETT E. E. E., JARRETT W. F. H., URQUHART : Quantitative studies on the kinetics of establishment and expulsion of intestinal nematode populations in susceptible and immune hosts Nippostrongylus brasiliensis in the rat. Parasitology, 1968, 58, 625-639.

Mogbel R., Denham D. A. : Strongyloides ratti : Parasitological observations on primary and secondary infections in the small intestine of rats. J. Helminthol., 1977, 51, 301-308.

Sheldon A. J. : Some experimental studies on Strongyloides ratti Am. J. hyg., 1937, 25, 39-65.

TALIAFERRo W. H., SARLES M. P. : The cellular reactions in the skin, lungs and intestine of normal and immune rats after infection with $N$. muris. J. Inf. Dis., 1939, 64, 157-192. 